\title{
MDM2 expression in EBV-infected nasopharyngeal carcinoma cells
}

\author{
Han-Chung $\mathrm{Wu}^{1,2}$, Tung-Ying Lu${ }^{1}$, Jeng-Jie Lee ${ }^{1}$, Jen-Kuo Hwang ${ }^{1}$, Yu-Ju Lin ${ }^{1}$, Chung-Kwe \\ Wang $^{1, *}$ and Chin-Tarng Lin ${ }^{1,3}$ \\ ${ }^{1}$ Institute of Pathology, ${ }^{2}$ Graduate Institute of Oral Biology, College of Medicine, National Taiwan University, \\ Taipei, Taiwan and ${ }^{3}$ Department of Pathology, National Taiwan University Hospital, Taipei, Taiwan
}

\begin{abstract}
To understand whether the p53-regulated $m d m 2$ gene expression was altered by the Epstein-Barr virus (EBV) in nasopharyngeal carcinoma (NPC), the NPC-TW01 cell line was infected by EBV through IgA receptor-mediated endocytosis. The $m d m 2$ gene was expressed only in a small fraction of the NPC cell population and could be enhanced in the EBV-infected $\left(\mathrm{EBV}^{+}\right)$cells. In the animals bearing EBV ${ }^{+}$and $\mathrm{EBV}^{-} \mathrm{NPC}^{-}$xenografts, the MDM2 ${ }^{+}$ cells only appeared in clusters in both $\mathrm{EBV}^{+}$and $\mathrm{EBV}^{-}$tumors with stronger expression in $\mathrm{EBV}^{+}$cells. Cotransfection of pmdm2-Luc plus pSV40-p53 plus pCMV-LMP1 in the NPC-TW06 line that had p53 heterozygous point mutation showed stronger $\mathbf{m d m} 2$ promoter activity than cells cotransfected with pmdm2Luc plus pSV40-p53, but no $\mathrm{mdm} 2$ promoter activity was seen in cells cotransfected with pmdm2-Luc plus pCMV-LMP1. Only the EBV-LMP1 but not the EBV-LMP2A gene could enhance p53 to upregulated mdm2 expression. Tumor cells in NPC biopsy specimens revealed similar $\mathrm{mdm} 2$ expression as in the animal model. It is concluded that although EBV can indirectly enhance $\mathbf{m d m} 2$ gene expression in tumor cells that express this gene, it cannot turn on or directly regulate $\mathbf{m d m 2}$ expression in cells that do not express this gene. In other words, EBV plays a role as an enhancer in NPC tumorigenesis.
\end{abstract}

Laboratory Investigation (2004) 84, 1547-1556, advance online publication, 27 September 2004; doi:10.1038/labinvest.3700183

Keywords: nasopharyngeal carcinoma; mdm2 expression; EBV infection; cotransfection of pmdm2-LuC; psv40p53; LMP1

Nasopharyngeal carcinoma (NPC) is a very common cancer in South China, Taiwan and Singapore. ${ }^{1}$ Although Epstein-Barr virus (EBV) has been closely associated with NPC, ${ }^{2,3}$ hereditary and environmental factors, such as salted fish, Chinese herbs and long-term exposure to sulfuric acid vapor, have also been suspected to be related to NPC induction. ${ }^{4,5}$ In fact, the etiological factors have yet to be clearly identified. In previous studies in which we attempted to investigate the molecular mechanism of NPC pathogenesis, we established nine NPC cell lines. ${ }^{6,7}$ Research findings on these cell lines and their original biopsy specimens indicate that EBV plays a major role in promoting tumor progression in NPC pathogenesis. ${ }^{8-12}$

In one of our experiments, ${ }^{12}$ we used severe combined immunodeficiency (SCID) mice to pro-

Correspondence: Dr C-T Lin, DDS, PhD, Department of Pathology, National Taiwan University Hospital, \#7, Chung-Shan S. Rd, Taipei 100, Taiwan.

E-mail: ctl@ha.mc.ntu.edu.tw

${ }^{*}$ Current address: Department of Internal Medicine, Ren-Ai Municipal Hospital, Taipei 106, Taiwan.

Received 29 June 2004; revised 13 August 2004; accepted 15 August 2004; published online 27 September 2004 duce $\mathrm{EBV}^{+}$and $\mathrm{EBV}^{-}$xenografts, in which certain oncogenes such as EGFR were only expressed in a fraction of $\mathrm{EBV}^{-}$tumor cells; EBV infection could enhance those cells to express the EGFR gene, but could not turn on the EGFR gene in other tumor cells which did not express this gene. ${ }^{12}$ However, this finding was a morphological observation. In order to dissect the molecular mechanism for this phenomenon, in the present study we used a cotransfection system to transfect the EBV-LMP1 gene, p53 gene and $m d m 2$ promoter into p53 mutated NPC cells to observe the actual relationship between EBV and $m d m 2$ gene expression, and also we compared the function of EBV-LAMP1 and EBV-LAMP2A genes in relation to $m d m 2$ gene expression.

In another study, we had investigated the p53 gene and its protein expression in our NPC cell lines. ${ }^{13}$ In that study we found a heterozygous point mutation at codon 280 in one of the NPC cell lines (NPC-TW06) similar to the reports from other laboratories. ${ }^{14,15}$ This cell line was used for cotransfection experiment. $m d m 2$ has been identified as an oncogene in the transgenic animal. It is the target gene of p53 protein. Its protein has been shown to negatively regulate $p 53$ function in other cellular 
systems. ${ }^{16-19}$ For these reasons, we also wanted to find out whether $m d m 2$ expression in NPC cell lines and biopsy specimens could be regulated by EBV infection in vitro and in vivo.

\section{Materials and methods}

\section{Cell Lines and Culture}

In these experiments, we used three representative NPC cell lines established in our laboratory (NPCTW01, 04 and 06), one derived from a keratinizing squamous cell carcinoma (NPC-TW01) ${ }^{14}$ and the other two (NPC-TW04 and 06) ${ }^{7}$ from undifferentiated carcinoma. Although the lines contained EBV DNA at early passages, they became negative after 30 passages. ${ }^{9}$ The cell lines we used had undergone 50 passages and showed no EBV signal. They were cultured in Dulbecco's modified Eagle's medium (DMEM) containing 5\% fetal calf serum (FCS) and incubated in a $10 \% \mathrm{CO}_{2}$ incubator. The other cell line, B95-8 line, a marmoset lymphoblastoid line containing latent EBV was used to isolate EBV particles and grown in RPMI-1640. This line was obtained from American Type Culture Collection.

\section{Preparation of NPC Biopsy Specimens}

A total of 20 NPC biopsy specimens were collected. One part of each specimen was fixed in formaldehyde for routine histopathalogical examination. The other part was directly put into a liquid nitrogen container for extraction of total RNA. In total, 15 of the specimens were identified as WHO type IIB (undifferentiated carcinoma), two as type I (keratinizing squamous cell carcinoma), and one as Type IIA (nonkeratinizing carcinoma).

\section{Preparation of EBV Particles for EBV Infection to NPC Cells}

The procedures used to isolate EBV particles and identify viral DNA in the isolated solution were performed in exactly the same way as in a recent report. ${ }^{12} \mathrm{EBV}$ infection was performed also according to the recent publication ${ }^{11,12,20}$ using IgA receptor (secretory component protein)-mediated endocytosis. After being infected, the cells were cultured for 10 days, collected and used for the detection of EBV genome by in situ PCR hybridization and EBNA-1 immunostaining, ${ }^{12}$ and subjected to the following experiments.

\section{Immunolocalization of MDM2 in $\mathrm{EBV}^{-}$and $\mathrm{EBV}^{+} \mathrm{NPC}$ Cells and NPC Biopsy Specimens}

For localization of MDM2 protein expression in $\mathrm{EBV}^{-}$and $\mathrm{EBV}^{+} \mathrm{NPC}$ cells, we used a routine immunohistochemical method previously deve- loped in our laboratory. ${ }^{12}$ Briefly, after EBV-infected NPC cells were incubated for 10 days, they and the $\mathrm{EBV}^{-}$cells were fixed for immunostaining with monoclonal antibodies against MDM2 (Oncogene Research Products, Boston, MA, USA) at 1:50 dilution. For comparison, we also performed immunostaining of MDM2 in NPC biopsy specimens using the same antibodies. Some normal mouse serum was used to replace the primary antibody as the negative control.

\section{Reverse Transcription (RT)-PCR Analysis of mdm2 RNA Expression in $\mathrm{EBV}^{-}$and $\mathrm{EBV}^{+}$NPC Cells and Biopsy Specimens}

To analyze the change of $m d m 2$ gene expression after NPC cells were infected with EBV and in 20 NPC biopsy specimens, we used a semi-quantitative RT-PCR to observe mdm2 mRNA expression as previously descripted. ${ }^{10}$ The primer sequences for the $m d m 2$ probe were adapted from a paper by Sigalas et al. ${ }^{19}$ They were L (sense) GCA GGG GAG AGT GAT ACA GAT and R (antisense) GAT GGC TGA GAA TAG TCT TCA containing $348 \mathrm{bp}$ in between two primers. For internal control, the actin primer sequences were used as published previously. ${ }^{10}$

\section{Western Blot Analysis of MDM2 in $\mathrm{EBV}^{-}$and $\mathrm{EBV}^{+}$ NPC-TW01 Cells and in Wild and Mutant p53 Gene Transfected NPC-TW01 Cells}

Wild-type (wt) and mutant-type (mt) p53 genes were cloned and subcloned into pGEM3 from the NPCTW06 cell line as described previously. ${ }^{5}$ Both genes were then transfected by lipofectamine separately into the NPC-TW01 cell line. They were transient transfected lines with a transfection efficiency of about $60 \%$, similar to that reported in one of our previous studies. ${ }^{13}$ The tumor cells from EBV ${ }^{-}$and $\mathrm{EBV}^{+}$NPC cells and from the wt and mt p53 transfected cells were then subjected to total protein extraction for Western blot analysis of MDM2 expression. $^{12}$ We used $\alpha$-tubulin as an internal control. The data were further analyzed by the densitometry.

\section{Animal Model Bearing $\mathrm{EBV}^{+}$and $\mathrm{EBV}^{-} \mathrm{NPC}^{\mathrm{Tumor}}$ Masses}

The $\mathrm{EBV}^{+}$and $\mathrm{EBV}^{-}$xenografts produced in SCID mice were obtained from the same animal experiments published in a recent paper $^{12}$ in which the SCID mice bearing $\mathrm{EBV}^{+}$xenograft grew faster and larger than SCID mice bearing $\mathrm{EBV}^{-}$xenograft. Some of the xenograft tissue fragments were fixed in formaldehyde and embedded in paraffin blocks for double localization of MDM2 and EBER-1 using the same technique described in the published paper. ${ }^{12}$ 


\section{Transient Cotransfection and Conditioned Media}

The transfection of different plasmids into NPCTW06 cells (with heterozygous mutation of p53 at codon 280) and NPC-TW04 cells (with wt p53) separately was carried out in the $6 \mathrm{~cm}$-diameter Petri dish containing $5 \times 10^{5}$ cells per dish with the use of Lipofectamine 2000 (Invitrogen) following the manufacturer's protocol. ${ }^{13,21}$ Each cell line was transfected with $3.8 \mu \mathrm{g}$ of appropriate reporter and effecter plasmids (including pGL2-mdm2-Luc, pGL3-SV40-p53 expression vector, $p$ GL3-CMV- $\beta$ gal, and pcDNA3.1-CMV-LMP1 and pRc/CMV-LMP2A. NPC cells for the transfection experiment were cultured in DMEM with 10\% fetal calf serum before the transfection was performed. During transfection, the cells were cultured in a serum-free medium (OPTI-MEM, GIBCO/BRL) without antibiotics for $4 \mathrm{~h}$ at $37^{\circ} \mathrm{C}$. After 2 days, the transfected cells were collected for luciferase assay. Transfection efficiency was normalized by cotransfection with a $\beta$-galactosidase reporter construct. The luciferase and galactosidase assays were performed according to the instructions in the commercial kits (Promega, MI, USA). Each transfection experiment was repeated four times.

\section{Colocalization of MDM2 and EBER-1, p53 and EBER-1,} MDM2 and p53 in NPC Biopsy Specimens

To colocalize MDM-2 or p53 with EBER-1 in paraffin sections ${ }^{12,13}$ immunostaining was first performed using either monoclonal antibody against MDM2 or rabbit polyclonal antibodies against p53 (prepared by our laboratory as described previously), ${ }^{13}$ and then followed by in situ nucleic acid hybridization of EBV with EBER-1 antisense probe, ${ }^{9,12}$ respectively. The substrate for immunostaining was peroxidase substrate, and the substrate for in situ hybridization was alkaline phosphatase (AP) substrate. In the double localization of MDM2 and p53, we used monoclonal antibodies against MDM2 plus AP-labeled second antibodies, and rabbit polyclonal antibodies against p53 plus peroxidase-labeled second antibodies. We used a sense probe to replace an antisense probe as an EBER-1 negative control, ${ }^{9}$ and used normal mouse serum and normal rabbit serum as the primary antibodies for negative immunostaining. No specific EBV signal could be detected when a sense probe was used, nor was immunostaining seen in the control normal serumtreated cells.

\section{Results}

Localization of MDM2 Protein in $\mathrm{EBV}^{-}$and $\mathrm{EBV}^{+}$ NPC-TW01 Cells

When NPC-TW01 cells were infected by EBV and in situ PCR hybridization and immunohistochemical localization using antibodies against EBNA-1 were performed, the infection efficiency was about 90\% (data not shown). When EBV ${ }^{-}$NPC-TW01 cells were stained with mAb against MDM2, a small subset of individual tumor cells showed weak to moderate and a few cells revealed strong anti-MDM2 immunostaining (Figure 1a: long arrow) in their nuclei, while a large number of tumor cells remained unstained (Figure 1a: short arrow). When $\mathrm{EBV}^{+}$cells (Figure 1b) were stained the same way, the reaction product in some small clones of tumor cells showed moderate to strong nuclear immunostaining (Figure 1b: long arrow). However, some NPC cells remained unstained (Figure 1b: short arrow). The MDM2-negative cells made up $65 \%$ of the $\mathrm{EBV}^{-}$cells and $68 \%$ of the $\mathrm{EBV}^{+}$cells. The percentages were obtained by taking averages after counting 15 high-power fields in each case (Figure 1a, b).

RT-PCR and Western Blot Analyses of mdm2 RNA and Protein Expressions in $\mathrm{EBV}^{-}$and $\mathrm{EBV}^{+} \mathrm{NPC}^{-}$Cell Lines

In RT-PCR, EBV ${ }^{+}$NPC-TW01 cells showed a stronger $348 \mathrm{bp}$ band of $\mathrm{mdm} 2$ signal (Figure 2a, lane 2) than that of the uninfected cells (Figure 2a, lane 1). Densitometry analysis revealed a 2.1-fold higher expression of $m d m 2$ in $\mathrm{EBV}^{+}$cells than in $\mathrm{EBV}^{-}$cells (Figure 2b). When EBV ${ }^{+}$and $\mathrm{EBV}^{-} \mathrm{NPC}$ cells were subjected to Western blotting a clear 90$\mathrm{kDa}$ band of MDM2 protein was seen in the EBV ${ }^{+}$ cells (Figure 2c, lane 2), while a rather weak MDM2 band was seen in the EBV ${ }^{-}$cells (Figure 2c, lane 1). Densitometry analysis revealed a 2.3-fold stronger expression of MDM2 protein in $\mathrm{EBV}^{+}$cells than in $\mathrm{EBV}^{-}$cells (Figure 2d, lanes 1, 2).

\section{Colocalization of MDM2 and EBER-1 in SCID Mice Bearing $\mathrm{EBV}^{+}$and $\mathrm{EBV}^{-} \mathrm{NPC}^{-}$Xenografts}

Double localization of EBER-1 and MDM2 in EBV ${ }^{-}$ xenografts showed no EBER-1 signal in the tumor cells (Figure 3b). However, clusters of tumor cells with moderate (with a few strong) MDM2 immunostaining were seen randomly distributed in the section (Figure $3 \mathrm{~b}$, large arrowhead). The tumor cells surrounding the clusters showed no immunoreactivity of anti-MDM2 (Figure 3b, small arrowhead). Double localization of EBER-1 and MDM2 in $\mathrm{EBV}^{+}$xenografts had different results. In the tumor sections, EBER-1 signals were seen in most tumor cells (Figure 3a, large and small white arrowheads). MDM2-positive cells were also seen in clusters in the tumor nests, but the staining intensity of MDM2 was very strong in those clusters in which many tumor cells revealed immunostaining in both the nucleus and cytoplasm (Figure 3a, large black arrowhead). Many cells surrounding the MDM2 ${ }^{+}$ clusters were $\mathrm{MDM}^{-}$and $\mathrm{EBV}^{+}$(Figure 3a, small white arrowhead). No EBV signal was seen in the 
negative control section; similarly, no immunostaining was shown in the nonspecific serum treated section (data not shown).
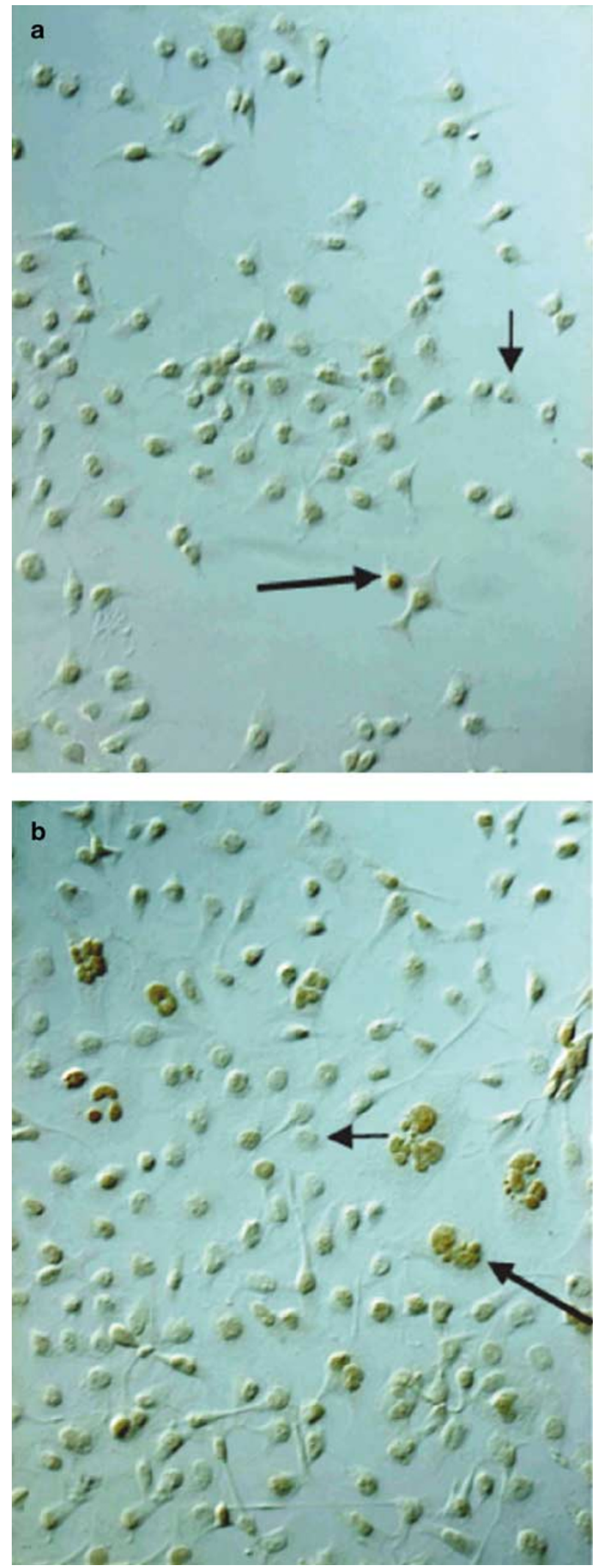

Western Blot Analysis of MDM2 in NPC-TW01 Cells that were Transfected with wt or mt p53 Genes Respectively

In Western blot analysis, one 90-kDa MDM2 band with a strong staining intensity was seen in the wt p53-transfected cells (Figure 4a, lane 1) and a weak MDM2 band was found in the mt p53-transfected cells (Figure 4a, lane 2). Densitometry analysis revealed a 2.1-fold higher intensity of wt p53 transfected band (Figure 4b, lane 1) than the MDM2 band in mt p53 transfected cells (Figure 4b, lane 2). The wt and $\mathrm{mt}$ p53 plasmids were all transiently transfected to the NPC-TW01 cells. We did not infect the wt p53-transfected NPC cells with

a
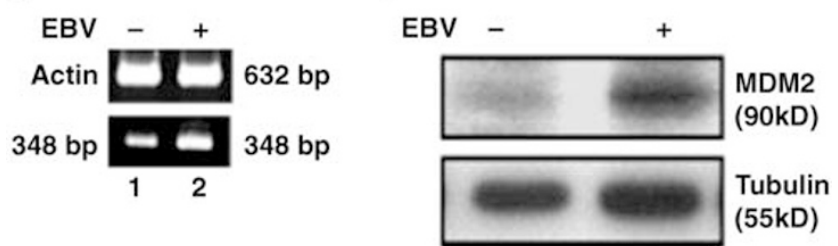

b

d
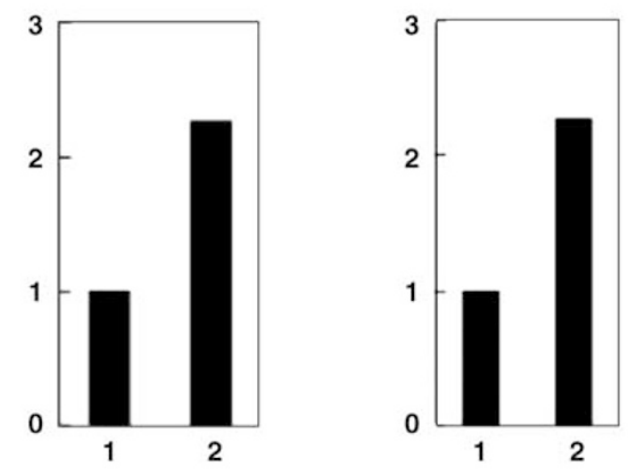

Figure 2 RT-PCR and Western blot analyses of $m d m 2$ gene and MDM2 protein expressions in NPC cell lines. (a) $m d m 2$ expression in $\mathrm{EBV}^{-}$and $\mathrm{EBV}^{+}$NPC-TW01 cell line. Lane 1: $\mathrm{EBV}^{-} \mathrm{NPC}$ cell line; lane 2: $\mathrm{EBV}^{+}$cell line. A weaker intensity of $348 \mathrm{bp}$ $m d m 2$ band is seen in lane 1 , while a stronger mdm2 band is shown in lane 2. The internal control of $632 \mathrm{bp}$ actin bands is also shown in both lanes. (b) Densitometry analysis shows a 2.3-fold higher $m d m 2$ expression in $\mathrm{EBV}^{+}$cells (lane 2) than in $\mathrm{EBV}^{-}$cells (lane 1). (c) Western blot analysis of MDM2 expression in $\mathrm{EBV}^{-}$ and $\mathrm{EBV}^{+}$NPC-TW01 cells. Lane 1, EBV ${ }^{-}$NPC cells; lane 2, $\mathrm{EBV}^{+}$NPC cells. A weaker band of $90 \mathrm{kDa}$ MDM2 protein is seen in lane 1, and a stronger band of $90 \mathrm{kDa}$ MDM2 protein is shown in lane 2. The internal control of $55 \mathrm{kDa}$ band of $\alpha$-tubulin in twolanes shows a similar staining intensity. (d) Densitometry analysis reveals a 2.2-fold higher MDM2 protein expression in $\mathrm{EBV}^{+}$cells (lane 2) than in $\mathrm{EBV}^{-}$cells (lane 1).

Figure 1 Immunostaining of MDM2 in $\mathrm{EBV}^{-}$and $\mathrm{EBV}^{+} \mathrm{NPC}$ cell lines: (a) $\mathrm{EBV}^{-}$NPC-TW01 cells; (b) $\mathrm{EBV}^{+}$NPC-TW01 cells. Immunoreactivity of anti-MDM2 is seen in the nuclei of a subset of tumor cells (long arrows) but other tumor cells show no reaction product (short arrow) in both (a) and (b). However, the intensity of immunostaining in the subset of $\mathrm{EBV}^{+}$cells (b) is stronger than that in the $\mathrm{EBV}^{-}$cells (a) and the MDM2 ${ }^{+}$cells appear in clusters in $(\mathbf{b}) .(\mathbf{a}, \mathbf{b}): \times 110$. 

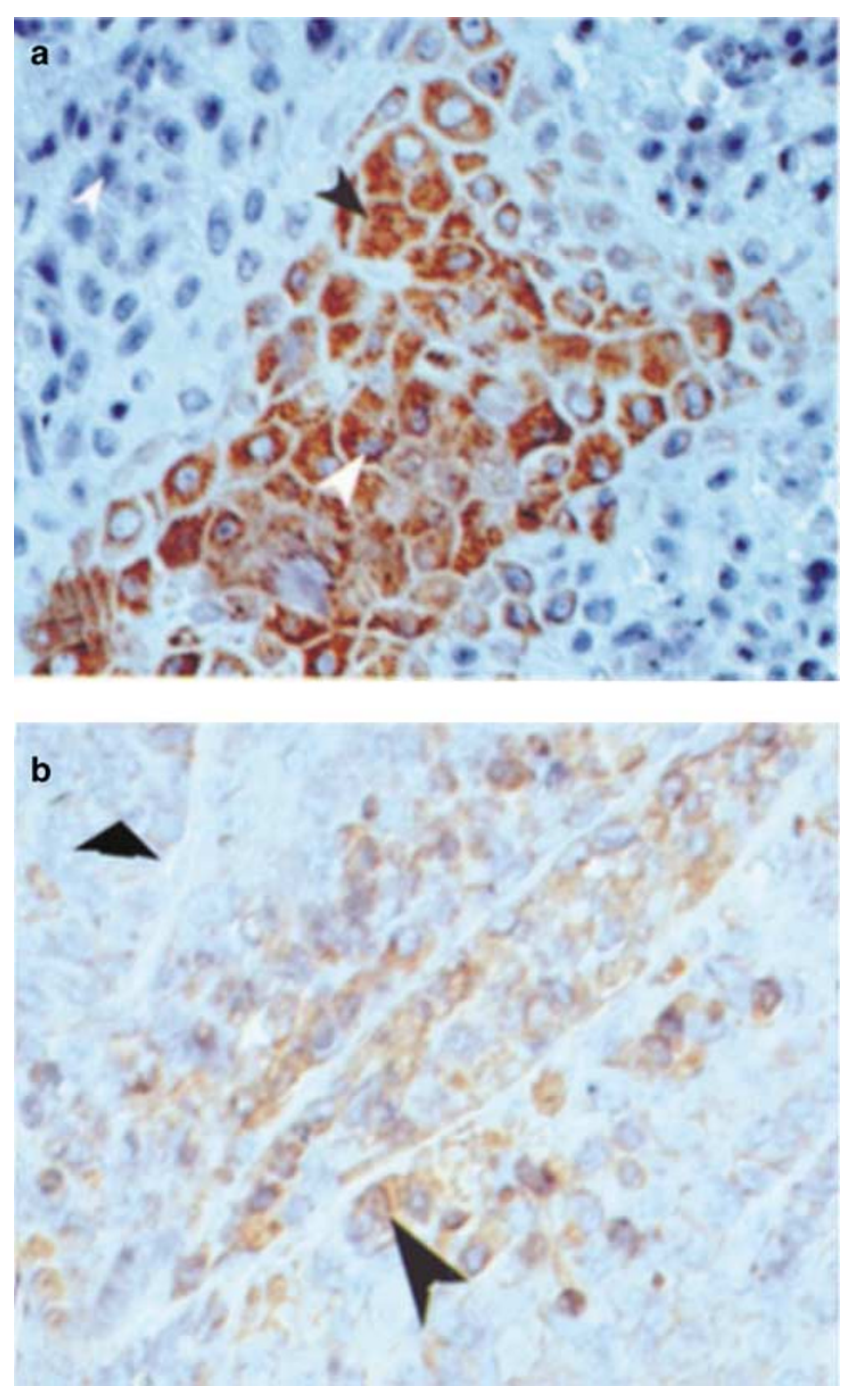

Figure 3 Colocalization of MDM2 and EBER-1 in SCID mice bearing $\mathrm{EBV}^{+}$and $\mathrm{EBV}^{-}$NPC-TW 01 xenografts: (a) $\mathrm{EBV}^{+} \mathrm{NPC}$ xenograft tumor section, (b) $\mathrm{EBV}^{-}$NPC xenograft section. (a) A cluster of tumor cells containing stronger MDM2 immunoreactivity (black arrowhead) and EBER-1 signal (large white arrowhead) is shown. The surrounding tumor cells contain clear EBER-1 signal (small white arrowhead) but no MDM2 reaction product. (b) No EBER-1 signal (small arrowhead) is seen in this EBV tumor section. But a cluster of tumor cells and some sporadic individual tumor cells (large arrowhead) reveal mild to moderate MDM2 immunoreactivity. While the surrounding tumor cells show no MDM2 reaction product (smaller arrowhead). (a, b): $\times 300$.

EBV to observe MDM2 expression, but we did perform an experiment using the cotransfection of a plasmid containing wt p53 and another plasmid containing $p L M P 1$ to NPC cells (as described in the following section).

Identification of $L M P 1$ Enhancement of p53 Activity to Activate the mdm2 Promoter by Cotransfection Experiments

Analysis of the activity of the $m d m 2$ promoter in NPC-TW06 line by transient transfection showed

\section{a} p53
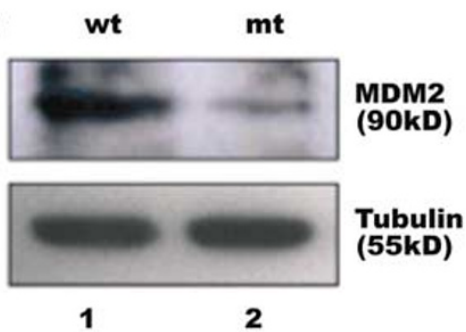

b

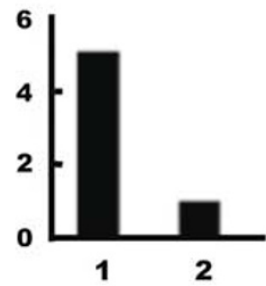

Figure 4 Western blot analysis of MDM2 protein in NPC-TW01 cells, which were transfected with wt and mt p53 genes separately. (a) Lane 1: NPC-TW01 cells transfected with wt p53 gene; lane 2: NPC-TW01 cells transfected with mt p53 gene. The $90 \mathrm{kDa}$ band of MDM2 in lane 1 is much stronger than that in lane 2 . The $55 \mathrm{kDa}$ band of $\alpha$-tubulin reveals similar staining intensity in both lanes 1 and 2. (b) Densitometry analysis reveals a 5.1-fold higher MDM2 protein expression in wt $p 53$-transfected NPC cells (lane 1) than mt p53-transfected cells (lane 2).

only background luciferase activity (Figure 5a, lane 1). If pmdm2-Luc was cotransfected with $p C M V$ $L M P 1$ (Figure 5a, lane 3 and $\mathrm{b}$, lane 2) or with pcDNA 3.1 (Figure 5a, lane 2; and b, lane 1) separately, only the background activity was seen again. However, when pGL3-SV40-p53 and pGL2-mdm2-Luc were cotransfected, a 2.6-fold increase over the vector control was seen (Figure 5a, lane 4). When pcDNA3.1-CMV-LMP-1 was cotransfected with pGL3-SV40-p53 and pGL2-mdm2-LuC, a 6.5-fold increase over the vector control could be found (Figure 5a, lane 5). If NPC cells containing a wt p53 gene, such as NPC-TW04 line, were cotransfected with pGL2-mdm2-Luc and pcDNA 3.1 , the endogenous p53 can spontaneously transactivate mdm2-Luc activity with a 2.1-fold over the NPC-06 cells (Figure 5b, lane 3). If the cells were also cotransfected with pGL2-mdm2-LuC and pcDNA3.1-CMV-LMP1, then pGL2-mdm2-Luc could be markedly upregulated with a 6.2-fold increase of Luc activity (Figure 5b, lane 4) in the presence of endogenous p53 protein. In order to verify whether LMP1 is the only EBV protein which can upregulate the expressed pGL2-mdm2-Luc, we also cotransfected a plasmid containing $L M P 2 A$ cDNA to the cells. Results showed that LMP2A could not upregulate expressed mdm2 expression directly or indirectly (Figure 5c, lanes 2 and 4).

\section{mdm2 Gene Expression in NPC Biopsy Specimens}

A total of 20 NPC biopsy specimens were used to examine $m d m 2$ mRNA expression. Results showed that $80 \%$ of NPC specimens appeared $m d m 2$ mRNA signals (Figure 6), though some had stronger and others had weaker signals. Densitometry analysis revealed that in some cases there were a 10-13-fold increase of $m d m 2$ expression (Figure 6, lanes 1, 4, 6 ), in other cases there were a 4-8-fold increase (Figure 6, lanes 2, 3, 5) when compared with the 
lowest amount of expression (Figure 6, lane 7), while in another case there was no $m d m 2$ expression (Figure 6, lane 8).

\section{Localization of MDM2 and p53 Protein in NPC Biopsy Specimens}

When 20 NPC paraffin sections were stained with antibodies against MDM2, one section was unreadable due to poor fixation and three showed no MDM2 immunostaining. The other 16 revealed sporadic tumor cells containing reaction product of anti-MDM2 in the nuclei. The distribution of positive cells was random and most tumor cells were unstained (Figure 7a). When stained by polyclonal anti-p53 antibodies, only a few cells in

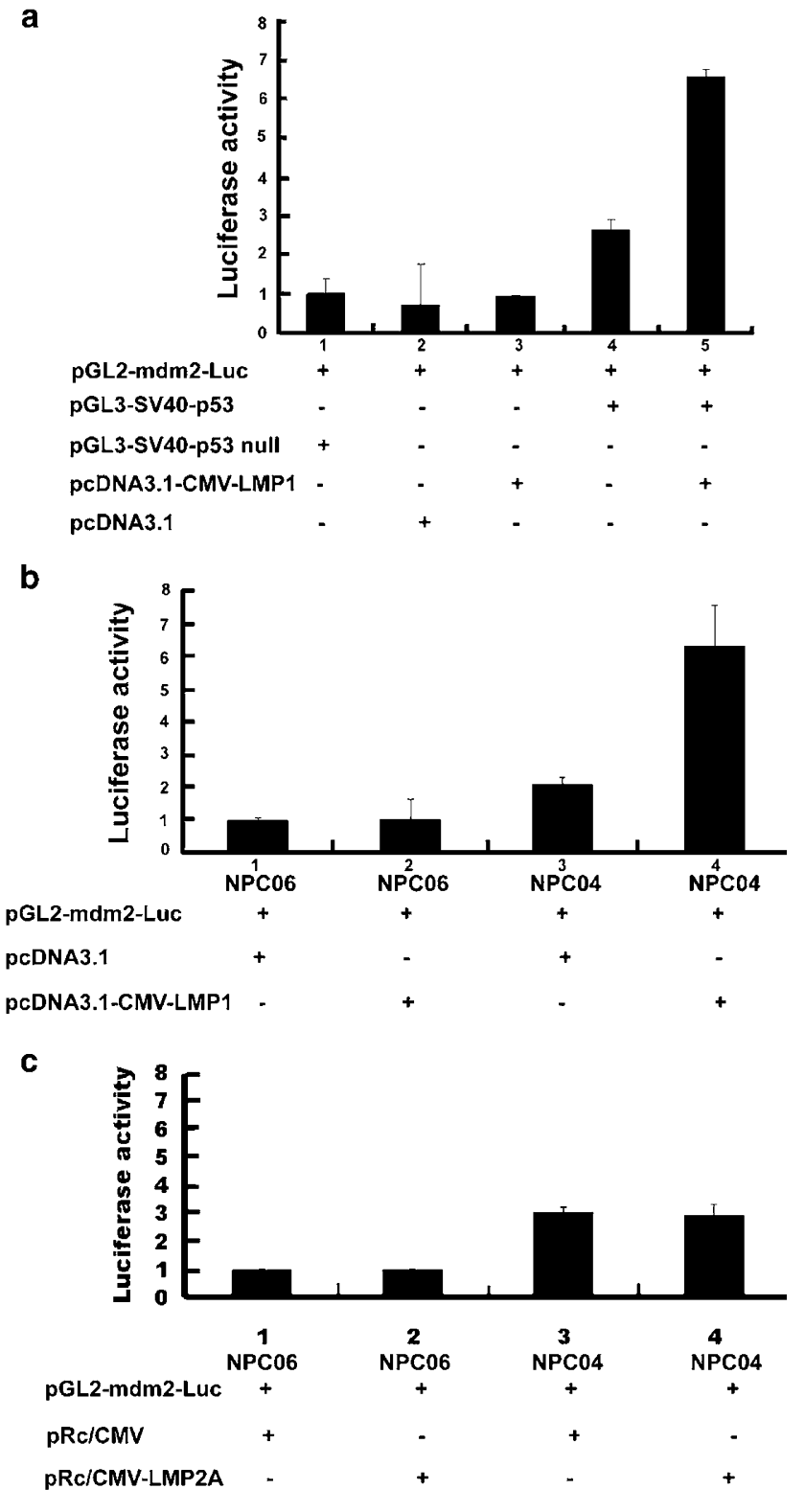

most NPC biopsy specimens showed nuclear staining, a few revealed more p53 stained cells than others (data not shown).

\section{a} Actin

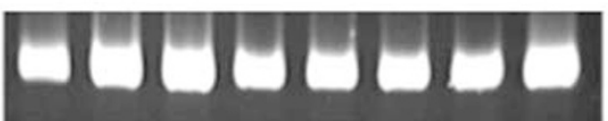
$632 b p$ $\mathrm{mdm} 2$

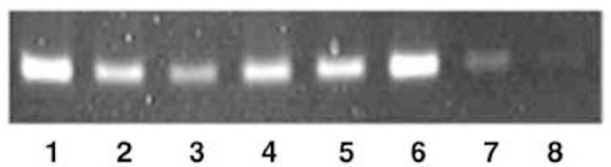
$348 \mathrm{bp}$

b

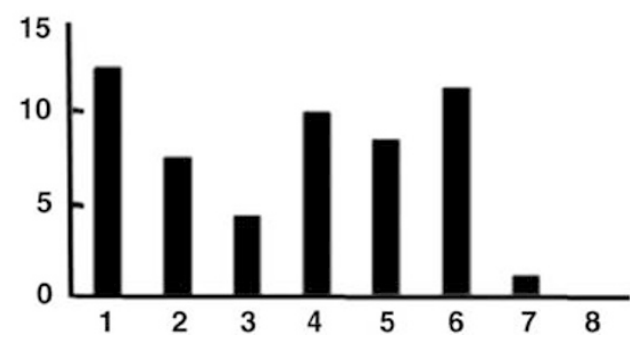

Figure 6 RT-PCR analysis of $m d m 2$ expression in NPC biopsy specimens. (a) $m d m 2$ expression in eight representative NPC biopsy tissue fragments were shown. Lanes 1-6 show a clear mdm2 band of $348 \mathrm{bp}$, while lane 7 shows a weak band and lane 8 reveals no $\mathrm{mdm} 2$ signal. The internal control $632 \mathrm{bp}$ band of actin is similar in each lane. (b) Densitometry analysis shows higher expression of $m d m 2$ mRNA in lanes 1-6. But lane 7 is very weak and lane 8 reveals no expression. The lowest expression intensity in the specimens (lane 7) was assigned as 1.

Figure 5 (a) Enhancement of EBV-LMP1 on p53 activation of mdm2 promoter in NPC-TW06 cells by cotransfection experiment. Lane 1: pGL2-mdm2-Luc plus pGL3-SV40-p53 null; lane 2: pGL2-mdm2-Luc plus pcDNA3.1; lane 3: pGL2-mdm2-Luc plus pcDNA3.1-CMV-LMP1; lane 4: pGL2-mdm2-Luc plus pGL3-SV40p53; lane 5: pGL2-mdm2-Luc plus pGL3-SV40-p53 plus pcDNA3.1-CMV-LMP1. Lanes 1-3 show only backgound luciferase activity; lane 4 shows an increased luciferase activity $(2.6 \times)$ higher than lane 1; lane 5 reveals a stronger luciferase activity $(6.5 \times)$ higher than lane 1. (b) Comparison of the effect of cotransfection of different plasmids in mt- and wt- p53 NPC cell lines. Lanes 1 and 2: NPC-TW 06 with p53 heterozygous point mutation; lanes 3 and 4: NPC-TW 04 with wt p53. Lanes 1 and 3: pGL2-mdm2-Luc plus pcDNA3.1 were cotransfected into NPCTW 06 and 04 separately; lanes 2 and 4: pGL2-mdm2-Luc plus pcDNA3.1-CMV-LMP1 were cotransfected into NPC-TW 06 and 04 separately. Lanes 1 and 2 shows only background luciferase activity; lane 3 reveals an increase of luciferase activity (2.2-fold) higher than lane 1; lane 4 shows a stronger luciferase activity (6.3fold) than lane 1. (c) Investigation of the effect of $L M P 2 A$ transfection. Lanes 1 and 3: NPC-TW 06 and 04 were cotransfected with pGL2-mdm2-Luc plus pRc/CMV separately; lanes 2 and 4: NPC-TW 06 and 04 were cotransfected with pGL2-mdm2$L u c+$ pRc/CMV-LMP2A separately. Both lanes 1 and 2 show background luciferase activity in p53 mt NPC-TW 06 line. But in NPC-TW 04 (containing wild type p53), in lanes 3 and 4, both lanes show similar luciferase activity higher than the background level due to endogenous p53 activation; LMP2A does not upregulate endogenous p53 gene to activate more mdm2luciferase activity. 

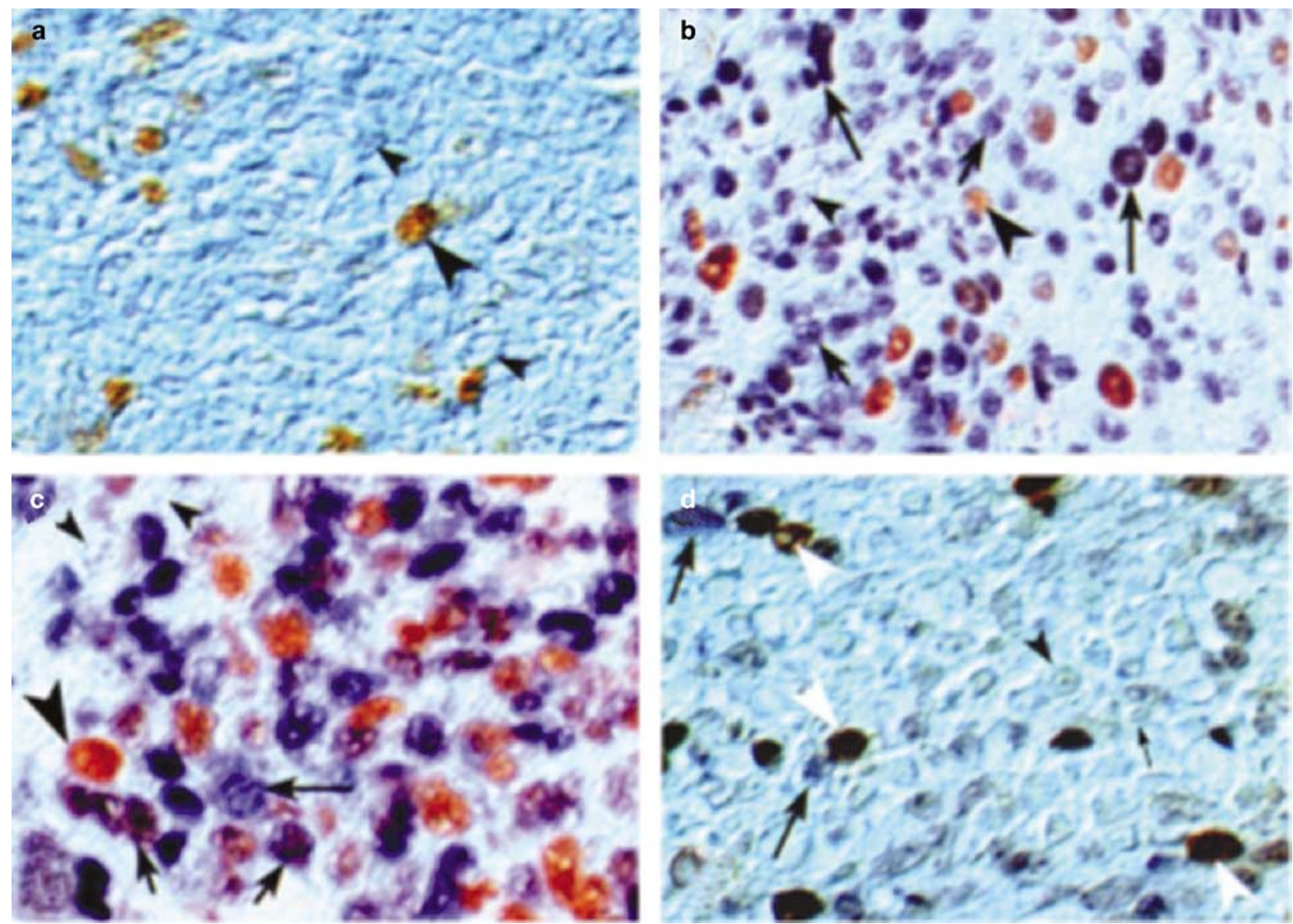

Figure 7 Immunolocalization of MDM2 protein and double localization of MDM2 and EBER-1, p53 and EBER-1, MDM2 and p53 in NPC biopsy specimens. Panels a-d were obtained from four different biopsy specimens. (a) Immunolocalization of MDM2 in NPC specimen. Only some tumor cells contain MDM2 reaction product (large arrowhead), many tumor cells are MDM2 negative (small arrowhead). (b) Double localization of MDM2 and EBER-1. The EBV signal (blue) is shown in many tumor cells (short arrows). Some tumor cells contain MDM2 reaction product (brown) only (large arrowhead). Other tumor cells reveal both MDM2 and EBV signal (purplish black color, long arrows). Small arrowhead: tumor cells without EBV or MDM2 protein. (c) Double localization of p53 protein and EBER-1 in an NPC biopsy specimen. It shows many EBV-positive cells (blue, long arrow) in the tumor section. Some EBV-positive cells also contain p53 protein (purplish black, short arrow). Other tumor cells either show p53 (brown, large arrowhead) only or contain neither p53 nor EBV (small arrowheads). (d) Colocalization of p53 and MDM2 in NPC biopsy specimens. Sections were double stained with rabbit anti-p53 (brown) and mAb anti-MDM2 (blue). Some tumor cells contain both p53 and MDM2 protein (large white arrowheads, purplish black color), while other cells show only MDM2 reaction product (large arrows). Very few or no tumor cell reveal(s) p53-positive cell only. Most other tumor cells were unstained (small arrowheads). A few possible lymphocytes with no EBER-1 signal are also visible (small arrow). $(\mathbf{a}-\mathbf{d}): \times 300$.

\section{Colocalization of MDM2 and EBER-1 in Biopsy Specimens}

In each of the 19 biopsy specimens, $\mathrm{EBV}^{+}$NPC cells (Figure 7b, short arrows, blue stained cells) were randomly distributed in the tumor and intermingled with $\mathrm{EBV}^{-}$and $\mathrm{MDM}^{-}$tumor cells (Figure 7b, small arrowhead), similar to previously described findings. ${ }^{9,11}$ In 16 cases, different degrees of nuclear staining of anti-MDM2 reaction product (brown) was seen in some $\mathrm{EBV}^{-}$cells (Figure 7b, large arrowhead, brown color) and in some $\mathrm{EBV}^{+}$cells (Figure 7b, long arrows, purplish black color); while three paraffin sections were $\mathrm{MDM}^{-}$. When calculating the number of $\mathrm{MDM}^{+}$only, $\mathrm{EBV}^{+}$only, and $\mathrm{MDM}^{+}$plus EBV ${ }^{+}$in the 19 biopsy specimens, we found that an average of $35 \%$ of the 19 biopsy specimens had only MDM2 ${ }^{+}$cells, $65 \%$ had only $\mathrm{EBV}^{+}$cells and $32 \%$ had MDM2 ${ }^{+}$plus EBV ${ }^{+}$(Table 1).When EBER-1 sense probe to replace the antisense probe was used to detect EBV in the sections, no specific EBV signal was seen (data not shown). When in situ PCR hybridization was used to detect EBV-DNA in the biopsy specimens, the result was the same as that of in situ hybridization of EBER-1 (data not shown), a result similar to our recent report. $^{12}$

\section{Colocalization of p53 and EBER-1 in NPC Biopsy Specimens}

Five out of the 18 readable specimens showed no p53 staining. The others revealed sporadic p53 
Table 1 Colocalization of MDM2 and EBER-1 in NPC specimens

\begin{tabular}{lcccccccccccccccccccccc}
\hline Human cases & 1 & 2 & 3 & 4 & 5 & 6 & 7 & 8 & 9 & 10 & 11 & 12 & 13 & 14 & 15 & 16 & 17 & 18 & 19 & 20 & Average $+(\%)$ \\
\hline MDM2(+) & $62^{\mathrm{b}}$ & 57 & 56 & 28 & 35 & 27 & 62 & 26 & 34 & 33 & 39 & 0 & 33 & 45 & 0 & 36 & 52 & 47 & 0 & $*$ & 35 & $*$ \\
EBER-1(+) & 67 & 77 & 58 & 49 & 59 & 55 & 74 & 57 & 70 & 63 & 65 & 61 & 60 & 76 & 68 & 64 & 63 & 77 & 63 & $*$ \\
MDM2(+)+EBER-1(+) & 44 & 53 & 43 & 26 & 33 & 26 & 58 & 26 & 33 & 33 & 38 & 0 & 33 & 44 & 0 & 35 & 44 & 41 & 0 & $*$ & 32 & 65 \\
\hline
\end{tabular}

${ }^{\mathrm{a}}$ The average number of stained cells was obtained from 19 biopsy specimens.

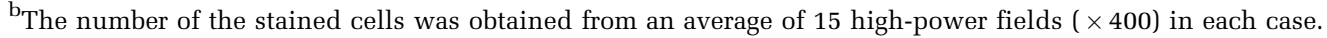

${ }^{*}$ Case 20 was fixed very poorly and unrecognizable; case 15 was also not fixed very well but still recognizable.

Table 2 Colocalization of p53 and EBER-1 in NPC specimens

\begin{tabular}{lrrrrrrrrrrrrrrrrrrrrrrr}
\hline Human cases & 1 & 2 & 3 & 4 & 5 & 6 & 7 & 8 & 9 & 10 & 11 & 12 & 13 & 14 & 15 & 16 & 17 & 18 & 19 & 20 & Average $+(\%)$ \\
\hline p53(+) & $0^{\mathrm{b}}$ & 17 & 0 & 27 & 19 & 12 & 18 & 0 & 43 & 15 & 47 & 68 & 35 & 30 & $*$ & 11 & 0 & 37 & 0 & $*$ & 21 \\
EBER-1(+) & 72 & 60 & 57 & 54 & 68 & 57 & 58 & 66 & 62 & 66 & 70 & 55 & 68 & 76 & $*$ & 70 & 64 & 81 & 69 & $*$ \\
p53(+)+EBER-1(+) & 0 & 12 & 0 & 23 & 15 & 10 & 16 & 0 & 29 & 9 & 37 & 39 & 28 & 22 & $*$ & 9 & 0 & 30 & 0 & $*$ & 16 & \\
\hline
\end{tabular}

${ }^{\mathrm{a}}$ The average number of stained cells was obtained from 18 biopsy specimens

${ }^{b}$ The number of the stained cells was obtained from an average of 15 high-power fields $(\times 400)$ in each case.

${ }^{*}$ Case 20 was fixed very poorly and unrecognizable; case 15 was also not fixed very well. After serial sections, it became unrecognizable.

stained cells. At higher magnification, the sections from some specimens showed a random distribution of $\mathrm{EBV}^{+}$cells without p53 staining (Figure 7c, long arrow, blue color), while in other tumor cells p53 staining was revealed in either $\mathrm{EBV}^{+}$cells (Figure 7c, short arrows, purplish-black) or $\mathrm{EBV}^{-}$cells (Figure 7c, large arrowhead, brown color). In addition, a few cells showed neither EBV nor p53 signal (small arrowheads). The average number of $\mathrm{p} 53^{+}$ cells was 21\%, EBER-1 65\%, and p53 ${ }^{+}$with EBER$1^{+} 16 \%$ (Table 2).

\section{Colocalization of MDM2 and p53 in Biopsy Specimens}

We selected four biopsy specimens to perform double localization of MDM2 and p53. The antip53 reaction product was brown, while the antiMDM2 reaction product was blue. We found certain tumor cells to be stained with both strong MDM2 and p53 reaction products (Figure $7 \mathrm{~d}$, large white arrowheads, brownish black). Only very rarely if at all did a tumor cell show p53 staining only, though some other tumor cells did show MDM2 reaction product only (Figure $7 \mathrm{~d}$, long arrows, blue color). Many tumor cells did not show either MDM2 or p53 staining (Figure 7d, small arrowhead). Some small cells, which might be tumor-infiltrating lymphocytes (short arrow), did not contain EBV signal. The average number of $\mathrm{MDM}^{+}$plus $\mathrm{p}^{+} 3^{+}$in the four selected cases was $29 \%$.

\section{Discussion}

In the present study, results from immunostaining, RT-PCR and Western blotting from EBV-infected cells all indicate that EBV can enhance mdm2 expression in a subset of cells that express this gene in vitro. This result is different from a previous report, ${ }^{18}$ in which no relationship was found between the presence of EBV and the expression of MDM2 protein in B lymphocytes. In order to determine whether these results would also be the same in vivo, we used SCID mice bearing $\mathrm{EBV}^{+}$and $\mathrm{EBV}^{-}$NPC-TW01 xenografts published in a recent paper. ${ }^{12}$ The findings that both $\mathrm{EBV}^{+}$and $\mathrm{EBV}^{-}$ xenografts containing clusters of tumor cells with MDM2 protein expression were surrounded by $\mathrm{MDM}^{-}$tumor cells (Figure 3 ) indicate that the MDM2 expressed NPC cells proliferate faster than the MDM2-negative cells and suggest that mdm2 gene has an oncogene like function. ${ }^{16,18,22}$ This finding was also supported by our in vitro cultured $\mathrm{EBV}^{+}$NPC cells, which showed stronger MDM2 expression cells grown in clusters (Figure 1). The fact that MDM2 expression was stronger in the $\mathrm{EBV}^{+}$mass than in the $\mathrm{EBV}^{-}$mass also indicates that EBV can enhance the MDM2 expression in the tumor cells that express this gene. Thus, the enhanced MDM2 expression in a subset of NPC cells after EBV infection may also play a role for EBV to promote tumor cell proliferation, as has been suggested previously. ${ }^{23,24}$ Since many $\mathrm{EBV}^{+}$and $\mathrm{EBV}^{-}$cells in mouse tumor masses also showed no MDM2 expression (Figure 3), EBV may not be able to turn on the $m d m 2$ gene expression in tumor cells that do not express MDM2 protein. In other words, EBV may only indirectly enhance MDM2 expression in tumor cells that express this protein but cannot turn on $m d m 2$ genes in cells that do not express this gene. This phenomenon seems to suggest that instead of EBV being a promoter able to turn on certain oncogenes or cell cycle regulatory factors to express their proteins in B lymphocytes in order to 
drive the $G_{0}$ phase cells getting into the cell cycle, ${ }^{3,24}$ it may be an enhancer only able to enhance the gene expression in the epithelial tumor cells that have already expressed this protein.

LMP1 has even been proposed in one report that in NPC it may contribute to invasiveness and metastasis through the induction of MMP-9 transcription and enzymatic activity. ${ }^{25}$ Since the authors in that report used a uterine cervical cancer cell line, which could express an MMP-9 transcript, it does not explain why EBV cannot induce MDM2 gene expression in the cells that do not express this gene, as shown in Figure 3a. In order to clarify this discrepancy, we used NPC-TW06 cell line, which has a heterozygous point mutation of p53 as mentioned above for our cotransfection experiment (Figure 5). Our results showed that LMP1 alone could not induce mdm2 promoter to express luciferase activity, yet the p53 protein could. In addition, LMP1 could enhance p53 activity to upregulate $m d m 2$ promoter. This conclusion was also confirmed by the other cotransfection experiment that in the NPC cell line containing wt p53 gene, only pcDNA3.1-CMV-LMP1 and pGL2-mdm2$L u c$ without exogenous p53 gene could upregulate mdm2-Luc activity (Figure 5b, lane 4). These findings support our hypothesis that EBV (LMP1) can indirectly enhance certain gene expressions in NPC cells that express those genes, but cannot induce gene expressions in cells that do not express them. In addition, another cotransfection experiment also indicates that it is EBV-LMP1 but not EBV-LMP2A gene can indirectly regulate expressed $m d m 2$ gene (Figure 5c). In one of our unpublished data, we have also transfected $E B N A$-1 gene to $\mathrm{EBV}^{+}$ NPC cells and showed no specific function of $E B N A$-1 gene to alter host gene expression, similar to $L M P-2 A$.

The findings that 16 out of 20 NPC cases contained weak to moderate $m d m 2$ expression, suggest that the expression of $m d m 2$ gene in different NPC tissue fragments is variable. The result that about $80 \%$ of NPC biopsy specimens have more or less expressed mdm2 mRNA is different from a previous report that $m d m 2$ gene was infrequently amplified or expressed in NPC. ${ }^{26}$ That only about $35 \%$ of tumor cells expressed MDM2 protein in each case (Table 1) suggests that overexpression of MDM2 protein in those tumor cells may occur during NPC tumor progression. Apparently, the mdm2 gene is not a 'gate keeper' gene for nasopharyngeal carcinoma. In addition, since certain MDM2-positive tumor cells were not infected by EBV (Figure 7), indicating that in vivo in NPC biopsy specimen $m d m 2$ gene expression in certain tumor cells is independent from EBV infection.

The fact that some tumor cells did contain both MDM2 protein and EBV signal suggests that EBV may enhance $m d m 2$ gene expression in these cells, a finding similar to the above-mentioned animal model. Our double localization of p53 and EBV in NPC biopsy specimens also demonstrated that some p53-containing cells may or may not be infected by EBV (Figure 7c), indicating that p53 expression in NPC biopsy specimen is also independent of EBV infection. But in the $\mathrm{EBV}^{+}-\mathrm{p} 53^{+}$cells, the $p 53$ gene expression can be enhanced by EBV infection (Figure 5a, lane 4).

mdm2 gene can be upregulated by p53 protein; it can also be regulated by the Ras-driven Raf/MEK/ MAP kinase pathway in a p53-independent manner. ${ }^{27,28}$ Whether this is also true in NPC is not clear. In the double localization of MDM2 and p53 in NPC biopsy specimens, only a fraction of MDM2-positive tumor cells contained p53 protein but most or all p53-positive cells contained MDM2 protein. This finding combined with the result from the wt p53 transfection experiment, which showed upregulation of $m d m 2$ gene expression, suggests that in wt p53-positive NPC tumor cells, p53 protein can up regulate MDM2 expression, but in MDM2-positive and p53-negative cells MDM2 expression is independent from p53 protein regulation. This finding supports the recent report that $m d m 2$ may be regulated by other signal pathways as mentioned above. The presence of MDM2 in those NPC tumor cells with negative p53 protein indicates that some functions of MDM2 are independent from p53 interaction, and may also have a role in promoting tumor growth $\mathrm{h}^{23-25}$ and increasing anchorage-independent growth ${ }^{29}$ in the pathogenesis of NPC. This conclusion is somewhat different from one previous study that has reported that mdm2 may deregulate the p53-dependent growth suppressive pathway in the pathogenesis of NPC. ${ }^{29}$

It is concluded that EBV plays a major role as an enhancer to the progression of NPC tumorigenicity and that EBV can enhance the expression of endogenous p53 and indirectly upregulate mdm2 expression only in the tumor cells that express MDM2 protein, but cannot turn on $m d m 2$ gene in cells that do not express this protein. In other words, EBV can indirectly enhance MDM2 expression through enhancement of p53 activation in a subset of NPC cells to promote $\mathrm{EBV}^{+}$tumor growth.

\section{Acknowledgements}

We thank Dr CS Shen of Department of Otolaryngology, National Taiwan University Hospital, for providing NPC biopsy specimens and Dr KZ Wu of Department of Genomic Medicine, National Taiwan University Hospital, for kindly providing pGL2mdm2-Luc, pGL3-SV-40-p53 and pGL3-CMV- $\beta$ Gal plasmids and Dr WB Wang and CH Tsai of Department of Microbiology, National Taiwan University, College of Medicine for providing pcDNA3.1-CMV$L M P 1$ and pRc/CMV-Lmp2A, respectively. This work was supported in part by grants from National Health Research Institute (NHRI-EX92-9014BL), 
National Science Council (NSC-91-2320-B-002-105 and NSC-93-3112-B-002-038), and a research grant from National Taiwan University Hospital (NTUH92-S57), Taipei, Taiwan to CTL.

\section{References}

1 Waterhouse L, Muir C, Shanmugaratnam K, et al. Cancer Incidence in Five Continents, International Agency for Research on Cancer Scientific Publication 42, Vol. IV. International Agency for Research on Cancer: Lyon, 1982.

2 Klein G, Giovanella BC, Lindahl $\mathrm{T}$, et al. Direct evidence for the presence of Epstein-Barr virus DNA and nuclear antigen in malignant epithelial cells from patients with poorly differentiated carcinoma of the nasopharynx. Proc Natl Acad Sci USA 1974;71: 4737-4741.

3 Niedobitek G, Hansmann ML, Herbst H, et al. EpsteinBarr virus and carcinoma: undifferentiated carcinoma but not squamous cell carcinomas of the nasopharynx are regularly associated with the virus. J Pathol 1991; 165:17-24.

4 Armstrong RW, Armstrong MJ, Yu CM, et al. Salted fish and inhalants as risk factor for nasopharyngeal carcinoma in Malaysian Chinese. Cancer Res 1983;43: 2967-2970.

5 Ho CK, Lo WCH, Huang PH, et al. Suspected nasopharyngeal carcinoma in three workers with long-term exposure to sulfuric acid vapor. Occup Environ Med 1999;56:426-428.

6 Lin CT, Wong CI, Chan WY, et al. Establishment and characterization of two nasopharyngeal carcinoma cell lines. Lab Invest 1990;62:713-724.

7 Lin CT, Chan WY, Chen W, et al. Characterization of seven newly established nasopharyngeal carcinoma cell lines. Lab Invest 1993;68:716-727.

8 Lin CT, Chen W, Hsu MM, et al. Clonal versus polyclonal Epstein-Barr virus infection in nasopharyngeal carcinoma cell lines. Lab Invest 1997;76: 793-798.

9 Lin CT, Dee AN, Chen W, et al. Association of EpsteinBarr virus, human papilloma virus and human cytomegalovirus in nine nasopharyngeal carcinoma cell lines. Lab Invest 1994;71:731-736.

10 Lin CT, Kao HJ, Lin JL, et al. Response of nasopharyngeal carcinoma cells to Epstein-Barr virus infection in vitro. Lab Invest 2000;80:1149-1160.

11 Lin CT, Lin CR, Tan GK, et al. The mechanism of Epstein-Barr virus infection in nasopharyngeal carcinoma cells. Am J Pathol 1997;150:1745-1756.

$12 \mathrm{Wu} \mathrm{HC}$, Lin YJ, Lee JJ, et al. Functional analysis of EBV in NPC cell. Lab Invest 2003;83:797-812.
13 Hwang JK, Lin CT. Colocalization of endogenous and exogenous p53 proteins in nasopharyngeal carcinoma cells. J Histochem Cytochem 1997;45:991-1003.

14 Effert P, McCoy R, Abdel-Hamid M, et al. Alterations of the p53 gene in nasopharyngeal carcinoma. J Virol 1992;66:3768-3775

15 Sun Y, Hegamyer G, Cheng YJ, et al. An infrequent point mutation of the p53 gene in human nasopharyngeal carcinoma. Proc Natl Acad Sci USA 1992; 89:6516-6520.

16 Lozano G, de Oca Luna RM. Minireview. MDM2 function. Biochim Biophys Acta 1998;1377, M 55-59.

17 Momand J, Wu HH, Dasgupta G. MDM2-master regulator of the p53 tumor suppressor protein. Gene 2000;242:15-29.

18 Piette J, Neel H, Marechal V. Mdm2: keeping p53 under control. Oncogene 1997;15:1001-1010.

19 Sigalas I, Calvert AH, Anderson JJ, et al. Alternatively spliced mdm2 transcripts with loss of p53 binding domain sequences: transforming ability and frequent detection in human cancer. Nat Med 1996;2: 912-917.

20 Sixbey JW, Yao QY. Immunoglobulin A-induced shift of Epstein-Barr virus tissue tropism. Science 1992;255: 1578-1580.

21 Sambrook J, Russell DW. Molecular Cloning. A laboratory Manual, Vol. 3, 3rd edn. Cold Spring Harbor Laboratory Press: Cold Spring Harbor, NY, 2001, Chapter $1 \mathrm{~b}$.

22 Teoh G, Urashima M, Ogata A, et al. MDM2 protein overexpression promotes proliferation and survival of multiple myeloma cells. Blood 1997;90:1982-1992.

23 Martin K, Trouche D, Hagemeier C, et al. Stimulation of E2F1/DP1 transcriptional activity by MDM2 oncoprotein. Nature 1995;375:691-694.

24 Xiao ZX, Chen J, Levine AJ, et al. Interaction between the retinoblastoma protein and the oncoprotein MDM2. Nature 1995;375:694-698.

25 Takeshita H, Yoshizaki T, Miller WE, et al. Matrix metalloproteinase 9 expression is induced by EpsteinBarr virus latent membrane protein 1c-terminal activation regions 1 and 2. J Virol 1999;73:5548-5555.

26 Qian W, Hu LF, Chen F, et al. Infrequent MDM2 gene amplification and absence of gross WAF1 gene alterations in nasopharyngeal carcinoma. Eur J Cancer B Oral Oncol 1995;31B:328-332.

27 Ries S, Biederer C, Woods D, et al. Opposing effects of Ras on p53: Transcriptional activation of mdm2 and induction of p19 ${ }^{\mathrm{ARF}}$. Cell 2000;103:321-330.

28 Zhou BP, Liao Y, Xia W, et al. HER-2/neu induces p53 ubiquitination via Akt-mediated MDM2 phosphorylation. Nat Cell Biol 2001;3:973-982.

29 Honda R, Yasuda H. Association of p19 (ARF) with Mdm2 inhibits ubiquitin ligase activity of Mdm2 for tumor suppressor p53. EMBO J 1999;18:22-27. 